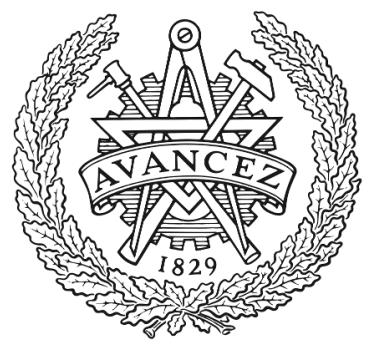

CHALMERS

UNIVERSITY OF TECHNOLOGY

\title{
Real-time-capable prediction of temperature and density profiles in a tokamak using RAPTOR and a first-principle-based transport model
}

Downloaded from: https://research.chalmers.se, 2023-04-26 14:30 UTC

Citation for the original published paper (version of record):

J Redondo, Meyer, H., Eich, T. et al (2018). Real-time-capable prediction of temperature and density profiles in a tokamak using RAPTOR and a first-principle-based transport model. Nuclear Fusion, 58(9). http://dx.doi.org/10.1088/1741-4326/aac8f0

N.B. When citing this work, cite the original published paper. 
Real-time-capable prediction of temperature and density profiles in a tokamak using RAPTOR and a first-principle-based transport model

To cite this article: F. Felici et al 2018 Nucl. Fusion 58096006

View the article online for updates and enhancements.
Optimal MSE polarisation angle and qprofile estimation using Kalman filters and the plasma simulator RAPTOB

M C C Messmer, F Felici, O Sauter et al.

Experimental validation of a Lyapunovbased controller for the plasma safety factor and plasma pressure in the TCV tokamak

B. Mavkov, E. Witrant, C. Prieur et al.

- First demonstration of real-time kinetic equilibrium reconstruction on TCV by coupling LIUQE and RAPTOR

F. Carpanese, F. Felici, C. Galperti et al. 


\title{
Real-time-capable prediction of temperature and density profiles in a tokamak using RAPTOR and a first-principle-based transport model
}

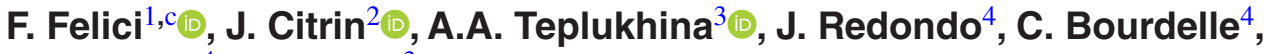 \\ F. Imbeaux ${ }^{4}$, O. Sauter ${ }^{3}$, JET Contributors ${ }^{a}$ and the EUROfusion MST1 \\ Team $^{\text {b }}$ \\ ${ }^{1}$ Department of Mechanical Engineering, Control Systems Technology Group, Eindhoven University \\ of Technology, PO Box 513, 5600MB, Eindhoven, Netherlands \\ ${ }^{2}$ DIFFER-Dutch Institute for Fundamental Energy Research, De Zaale 20, 5612AJ Eindhoven, \\ Netherlands \\ 3 Ecole Polytechnique Fédérale de Lausanne (EPFL), Swiss Plasma Center (SPC), CH-1015 Lausanne, \\ Switzerland \\ ${ }^{4}$ CEA, IRFM, F-13108 Saint Paul Lez Durance, France \\ E-mail: federico.felici@epfl.ch
}

Received 27 November 2017, revised 30 April 2018

Accepted for publication 31 May 2018

Published 3 July 2018

\section{Abstract}

The RAPTOR code is a control-oriented core plasma profile simulator with various applications in control design and verification, discharge optimization and real-time plasma simulation. To date, RAPTOR was capable of simulating the evolution of poloidal flux and electron temperature using empirical transport models, and required the user to input assumptions on the other profiles and plasma parameters. We present an extension of the code to simulate the temperature evolution of both ions and electrons, as well as the particle density transport. A proof-of-principle neural-network emulation of the quasilinear gyrokinetic QuaLiKiz transport model is coupled to RAPTOR for the calculation of first-principle-based heat and particle turbulent transport. These extended capabilities are demonstrated in a simulation of a JET discharge. The multi-channel simulation requires $\sim 0.2 \mathrm{~s}$ to simulate 1 second of a JET plasma, corresponding to $\sim 20$ energy confinement times, while predicting experimental profiles within the limits of the transport model. The transport model requires no external inputs except for the boundary condition at the top of the $\mathrm{H}$-mode pedestal. This marks the first time that simultaneous, accurate predictions of $T_{\mathrm{e}}, T_{\mathrm{i}}$ and $n_{\mathrm{e}}$ have been obtained using a first-principlebased transport code that can run in faster-than-real-time for present-day tokamaks.

Keywords: integrated tokamak simulation, real-time control, machine learning, tokamak profiles, tokamak transport

(Some figures may appear in colour only in the online journal)

\footnotetext{
${ }^{\text {a }}$ See the author list of [47].

${ }^{\mathrm{b}}$ See the author list of [48].

${ }^{c}$ Present address: SPC/EPFL, Lausanne, Switzerland.
} 


\section{Introduction}

For future, larger tokamaks, as well as for reactor applications, it will be increasingly important to thoroughly prepare and optimize discharges before they are executed. This requires simulation tools that can accurately and rapidly simulate the time-evolution of the plasma. In addition, testing, preparation and verification of plasma control systems benefit from tokamak simulation models that can execute in real-time [1]. For such purposes, it is beneficial to have a fast, controloriented simulation code, capable of evolving the quantities of interest as rapidly as possible.

One control-oriented simulator for the core plasma transport evolution is the RAPTOR code. Presently, the code has two main applications. Used off-line, it acts as a fast transport simulator capable of simulating an entire plasma discharge in a few seconds on a single core of a standard PC. This makes it highly suited for discharge analysis and preparation, as well as automated optimization of plasma discharges [2-4], or for design of feedback controllers $[5,6]$. Other codes aimed at fast tokamak simulation for this application include the METIS code, part of the CRONOS suite [7] and the control-designoriented transport simulator developed at Lehigh University [8].

Another important application of a control-oriented simulator, which is so far unique to RAPTOR, is its use as part of a tokamak plasma control system. In this application, it is used in conjunction with a state observer algorithm, specifically an Extended Kalman Filter [9], to merge diagnostic measurements with model-based prediction in real-time. Additionally, real-time predictions of the expected plasma evolution, also calculated in the control system, can be used for real-time monitoring of the plasma performance. In this function, RAPTOR has been implemented in the control systems of TCV [10], ASDEX-Upgrade [11] and RFX [12].

The version of RAPTOR used so far was capable of simulating the coupled evolution of poloidal magnetic flux (current diffusion) and electron temperature $T_{\mathrm{e}}$, requiring user-defined assumptions on the other profiles. In this paper we present the extension of the code to allow simultaneous simulations of $T_{\mathrm{e}}$, $T_{\mathrm{i}}, n_{\mathrm{e}}$ and other particle density species, and demonstrate for the first time the capability to predict core kinetic profiles in real-time.

When predicting the evolution of temperature and particle density, the result strongly depends on the thermal conductivity and particle diffusion and pinch terms. Direct numerical simulation of turbulent fluxes within a nonlinear gyrokinetic framework (e.g. GENE [13] or GYRO [14]) shows increasingly routine agreement between predictions and experiments. However, the computational cost $-\sim 10^{4}$ CPUh for fluxes at a single radius for a single time point, at ion-scales only-is prohibitive for routine profile prediction applications.

The quasilinear approximation for transport prediction provides significant computational speed-up, while still reproducing nonlinear simulations over a wide range of parameter space. Examples are QuaLiKiz (see [15, 16], and references therein), and TGLF $[17,18]$. These transport models are presently used routinely for scenario interpretation and prediction in integrated modelling. However, the computation cost is then still a few seconds for fluxes at a single radius, corresponding to $~ 50$ CPUh per second of simulated plasma evolution for JET. While 6 orders of magnitude faster than nonlinear simulations, it is still too slow for real-time computation.

Therefore, earlier versions of RAPTOR used empirical models for the thermal conductivity to predict the temperature profile evolution. By empirically tuning the various parameters of the transport models for a given operational regime, experimental results could be adequately matched, for example simulating entire discharges for ASDEX-Upgrade and TCV [4]. For applications in real-time control design for a particular experiment, with a known plasma scenario, this was sufficient. However, if we wish to predict the core profiles in a wide range of the operational space, for several devices and for future tokamaks without having to re-tune such parameters, transport models with better predictive capabilities are required, while still being sufficiently fast to be used in control-oriented applications.

One way to achieve this is to use neural networks to emulate the results of first-principle-based turbulent transport predictions. Model output databases are compiled with inputs spanning a portion of the experimentally relevant parameter space. The neural network then learns the mapping from code inputs to outputs. The speed of the quasilinear models allows for the production of sufficiently large training sets for this application. A first demonstration of this approach was shown in [19], where QuaLiKiz was emulated within a restricted 4D input space, where each input was varied over an experimentally relevant range (corresponding to $\sim 10^{5}$ points in total). While useful as a demonstration, the version of the transport model used in [19] assumed adiabatic electrons and returned only electron heat transport coefficients. In the present paper, we have extended this proof-of-principle neural network transport model to include kinetic electrons. This updated transport model-named QLKNN-4Dkinnow predicts both electron and ion heat fluxes, as well as electron particle diffusion and pinch terms. Impurity transport is not included in this proof-of-principle model. More recently, a neural network was used to emulate TGLF outputs, using higher input dimensionality (23D), but restricted to input parameter combinations encountered in a set of 24 discharges from the DIII-D tokamak [20].

Another important bottleneck in integrated transport simulation is the self-consistent calculation of the various sources of particles, power and auxiliary current, which often take a significant fraction of the simulation time. When performing rapid simulations (for optimization or control purposes, as is often the case or RAPTOR), results from earlier runs of physics-based models can be directly used, or parametrized. Alternatively, real-time-capable source codes can be used, such as real-time TORBEAM for Electron Cyclotron Heating/ Current drive [21, 22], and the recently developed RABBIT code [23] for Neutral Beam Injection. When it is not possible to make real-time capable physics models, a neural network emulation can also be carried out. In the work presented here, source profiles are directly taken from previous runs of more sophisticated codes. 
In this paper, we demonstrate the use of QLKNN-4Dkin in the extended RAPTOR-code to predict $T_{\mathrm{e}}, T_{\mathrm{i}}, q$ and $n_{\mathrm{e}}$ simultaneously for a JET discharge. We obtain core profiles that agree with results from the CRONOS transport simulation suite [7] using the same transport model, confirming that the equations are solved correctly in RAPTOR. These profiles reasonably predict the experimental measurements. Remaining discrepancies can be understood from the limits of the transport model used. We stress that the transport model does not have any free parameters other than the boundary conditions set at the top of the pedestal. The RAPTOR simulations run faster than real-time on a standard laptop $(2.6 \mathrm{GHz}$ Intel ${ }^{\mathrm{R}}$ core $^{\mathrm{TM}} \mathrm{i5}$ ), taking approximately $1 \mathrm{~s}$ to simulate $5 \mathrm{~s}$ of JET discharge with time steps of $0.1 \mathrm{~s}$.

The remainder is organised as follows: section 2 details the multichannel transport equations solved within RAPTOR, and introduces the QLKNN-4Dkin transport model. Section 3 shows the results of the JET simulation and comparison with CRONOS. Conclusions and an outlook to future work is provided in section 4 .

\section{RAPTOR transport simulations with multiple channels}

In this section we list the equations solved in RAPTOR as well as the main assumptions in the code. We also describe the QLKNN-4Dkin transport model in further detail.

\subsection{Equations solved in RAPTOR}

2.1.1. Basic assumptions and coordinate conventions. The RAPTOR code assumes an axisymmetric tokamak equilibrium with right-handed $(R, \phi, Z)$ cylindrical coordinate system, poloidal flux defined as $\psi(R, Z)=\int_{S} \mathbf{B}_{\mathrm{p}} \cdot \mathrm{d} \mathbf{S}_{\mathbf{z}}$, and $I_{\mathrm{p}}$ and $B_{0}$ always positive ${ }^{5}$.

In all equations, the normalized square root of the toroidal flux $\hat{\rho}=\rho_{\text {tor }, N}$ is used as the spatial variable, defined as $\hat{\rho}=\sqrt{\Phi / \Phi_{b}}$ with $\Phi(\psi)=\int_{S_{\psi}} B_{\phi} \mathrm{d} S_{\phi}$ the toroidal magnetic flux enclosed by a poloidal flux surface, and $\Phi_{b}$ denotes the toroidal flux enclosed by the last closed flux surface (LCFS). We write separate equations for $\psi$ and generic equations for energy and particle transport for each species, as follows.

2.1.2. Flux diffusion equation. This is the parabolic PDE used to evolve the distribution of poloidal magnetic flux $\psi(\rho, t)$ in time:

$$
\begin{aligned}
\sigma_{\|}\left(\left.\frac{\partial \psi}{\partial t}\right|_{\hat{\rho}}-\frac{\hat{\rho} \dot{\Phi}_{b}}{2 \Phi_{b}} \frac{\partial \psi}{\partial \hat{\rho}}\right)= & \frac{F^{2}}{16 \pi^{2} \mu_{0} \Phi_{b}^{2} \hat{\rho}} \frac{\partial}{\partial \hat{\rho}}\left[\frac{g_{2} g_{3}}{\hat{\rho}} \frac{\partial \psi}{\partial \hat{\rho}}\right] \\
& -\frac{B_{0}}{2 \Phi_{b} \hat{\rho}} V_{\hat{\rho}}^{\prime}\left(j_{b s}+j_{\text {aux }}\right)
\end{aligned}
$$

$\sigma_{\|}$is the neoclassical conductivity, for which we use the expression in the Sauter model $[25,26] . j_{\text {aux }}=\left\langle\mathbf{j}_{\text {aux }} \cdot \mathbf{B}\right\rangle / B_{0}$

${ }^{5}$ In the COCOS tokamak coordinate convention system [24], RAPTOR has $\operatorname{COCOS}=11$, the same as the ITER convention and with $\sigma_{I_{\mathrm{p}}}=\sigma_{B_{\phi}}=1$. is the non-inductive current density driven by auxiliary systems. The bootstrap current density $j_{b s}=\left\langle\mathbf{j}_{\text {bs }} \cdot \mathbf{B}\right\rangle / B_{0}$ is also computed from the Sauter model. $F=R B_{\phi}, V_{\hat{\rho}}^{\prime}=\frac{\partial V}{\partial \hat{\rho}}$, $g_{2}=\left\langle\left|\nabla V^{2}\right| / R^{2}\right\rangle$ and $g_{3}=\left\langle 1 / R^{2}\right\rangle$ (where $\langle\cdot\rangle$ denote the fluxsurface average), are terms which depend on the magnetic equilibrium.

The boundary condition at the magnetic axis $\hat{\rho}=0$ is $\frac{\partial \psi}{\partial \hat{\rho}}=0$ while at the plasma boundary $\hat{\rho}=1$ we use the relation $\frac{g_{2} g_{3}}{\hat{\rho}} \frac{\partial \psi}{\partial \hat{\rho}}=\frac{16 \pi^{3} \mu_{0} \Phi_{b}}{F}$ to prescribe a Neumann boundary condition for $\frac{\partial \psi}{\partial \hat{\rho}}$ via the total plasma current $I_{\mathrm{p}}$ at the plasma edge.

2.1.3. Energy transport equations. We write the energy transport equation, from [27], for a generic species $s$ as:

$$
\begin{aligned}
& \frac{3}{2}\left(V_{\hat{\rho}}^{\prime}\right)^{-5 / 3}\left(\left.\frac{\partial}{\partial t}\right|_{\hat{\rho}}-\frac{\dot{\Phi}_{b}}{2 \Phi_{b}} \frac{\partial}{\partial \hat{\rho}} \hat{\rho}\right) \\
& +\left[\left(V_{\hat{\rho}}^{\prime}\right)^{5 / 3} n_{\mathrm{s}} T_{\mathrm{s}}\right]+\frac{1}{V_{\hat{\rho}}^{\prime}} \frac{\partial}{\partial \hat{\rho}}\left(-\frac{g_{1}}{V_{\hat{\rho}}^{\prime}} n_{\mathrm{s}} \chi_{\mathrm{s}} \frac{\partial T_{\mathrm{s}}}{\partial \hat{\rho}}+\frac{5}{2} T_{\mathrm{s}} \Gamma_{\mathrm{s}} g_{0}\right)=P_{\mathrm{s}}
\end{aligned}
$$

where $T_{\mathrm{s}}(\rho, t)$ and $n_{\mathrm{s}}(\rho, t)$ are the temperatures and densities of the considered species. $\chi_{\mathrm{s}}$ is the thermal diffusivity and $\Gamma_{\mathrm{s}}$ is the convective flux (4). $P_{\mathrm{s}}$ is the sum of power density sources and sinks, which will be discussed later. $g_{0}=\langle|\nabla V|\rangle$ and $g_{1}=\left\langle|\nabla V|^{2}\right\rangle$ are again flux-surface averaged terms that depend on the magnetic equilibrium geometry. In practice, (2) is re-written as an equation for $T_{\mathrm{s}}(\rho, t)$ where $n_{\mathrm{s}}(\rho, t)$ is considered an external parameter entering into the equation. To obtain a unique solution, the value of the plasma temperature is prescribed at the plasma boundary or at another radial location close to the edge. This is discussed in more detail in appendix.

2.1.4. Particle transport equations. The particle transport equation, again following [27], is written for the species $s$ as:

$$
\frac{1}{V_{\hat{\rho}}^{\prime}}\left(\left.\frac{\partial}{\partial t}\right|_{\hat{\rho}}-\frac{\dot{\Phi}_{b}}{2 \Phi_{b}} \frac{\partial}{\partial \hat{\rho}} \hat{\rho}\right)\left[\left(V_{\hat{\rho}}^{\prime}\right) n_{\mathrm{s}}\right]+\frac{1}{V_{\hat{\rho}}^{\prime}} \frac{\partial}{\partial \hat{\rho}} \Gamma_{\mathrm{s}}=S_{\mathrm{s}}
$$

with

$$
\Gamma_{\mathrm{s}}=-\frac{g_{1}}{V_{\hat{\rho}}^{\prime}} D_{\mathrm{s}} \frac{\partial n_{\mathrm{s}}}{\partial \hat{\rho}}+g_{0} V_{\mathrm{s}} n_{\mathrm{s}}
$$

where $D_{\mathrm{s}}$ ad $V_{\mathrm{s}}$ are the particle diffusivity and pinch terms (positive $V_{\mathrm{s}}$ corresponds to outward pinch) and $S_{\mathrm{s}}$ are (spatially distributed) sources of particles. Similarly to the energy transport equation, boundary conditions can be given at the plasma boundary or another radial location close to the edge (see appendix).

After choosing for which species to solve the transport PDE above, densities of other species and the effective charge are constrained by the quasineutrality condition $n_{\mathrm{e}}=\sum_{j} Z_{j} n_{j}$ (where $Z_{j}$ is the charge of the $j$ th ion species) and $n_{\mathrm{e}} Z_{\mathrm{eff}}=\sum_{j} Z_{j}^{2} n_{j}$. If $Z_{\mathrm{eff}}$ is prescribed as input to the code then two particle densities can be determined. If $Z_{\text {eff }}$ is not prescribed, then only one particle density can be constrained 
and the others have to be manually prescribed or solved for. Typically one solves for either electrons or main ions. Separate transport of impurity species can in principle be simulated as well but is outside the scope of the transport model applied in this work.

\subsection{Treatment of magnetic geometry, sources, and MHD}

2.2.1. Treatment of magnetic geometry. The (possibly timevarying) terms in the equations that depend on the magnetic geometry $\left(g_{0}, g_{1}, g_{2}, g_{3}, F, V_{\hat{\rho}}^{\prime}\right)$ are assumed to be externally prescribed, as explained in detail in [4]. An external GradShafranov code is used to compute the flux-surface averages for the required times and the results are passed as input to the code. Iterative coupling with a fixed-boundary equilibrium code has been achieved but this is not used in the present paper. All simulations shown in this paper are done with a fixed equilibrium geometry.

2.2.2. Sources and sinks. Heating and current drive sources and sinks are either externally prescribed (as $\hat{\rho}$-dependent heat deposition/current density profiles from more sophisticated physics-based source simulation codes), or calculated using simple Gaussian profiles, prescribing deposition location and width, as in [2]. For real-time applications, results from real-time ray tracing calculations (e.g. TORBEAM [22]) can be used. Other sources and sinks are modeled as described in [2]. In the simulations shown in this paper, ohmic power, electron-ion equipartition and bremsstahlung are selfconsistently calculated. NBI and ICRH power are taken from physics-based codes, as will be detailed later. Line-radiation is included based on experimentally-estimated profiles.

2.2.3. Sawteeth and NTMs. RAPTOR includes modules for simulating sawtooth crashes and Neoclassical Tearing Modes. The sawtooth module is discussed in [12], and uses the Porcelli crash criterion for determining the onset of a sawtooth crash. A Kadomtsev full reconnection model is used for computing the new profiles of magnetic flux, temperature, and particle density following the sawtooth crash [28]. Neoclassical tearing modes are simulated self-consistently by using the generalized Rutherford equation to evolve the width of the island, while increasing the thermal transport coefficient in the region of the island to simulate the effect of enhanced transport across flux surfaces reconnected by the island [29, 30].

\subsection{Transport coefficients for particles and energy}

Solving (2) and (3) requires expressions for $\chi_{\mathrm{s}}, D_{\mathrm{s}}$ and $V_{\mathrm{s}}$ for all the species solved. In RAPTOR, several transport models can be chosen. Empirical transport models for the thermal diffusion can be used, such as Bohm-gyroBohm [31], as well as the empirical expression introduced in [2] to simulate improved confinement at low magnetic shear. More recently, a more advanced critical-gradient-based empirical model was introduced [4], which requires prescribing the $\mathrm{H}$-factor and the line-averaged density of the discharge as a function of time. In the present work, we use a novel neural-network emulation of results of the QuaLiKiz quasilinear gyrokinetic transport model, which is described in more detail below.

\subsubsection{Neural-network emulation of the quasilinear gyrokinetic} transport model QuaLiKiz. As described in the introduction, QLKNN-4Dkin is a neural network regression of the QuaLiKiz model in a restricted input dimensionality subspace using a multilayer perceptron network [32]. A first proof-ofprinciple version of this model was presented in [19], which used adiabatic electrons and only predicted electron heat conductivity. The version used for the present paper has been extended by including kinetic electrons and allows simultaneous evaluation of ion and electron heat conductivities, and electron particle diffusivity and pinch. The training set consists of a database of $\sim 10^{5}$ fluxes, generated using QuaLiKiz, perturbing 4 inputs $\left(R / L_{T_{\mathrm{i}}}, T_{\mathrm{i}} / T_{\mathrm{e}}, q, \hat{s}\right)$, covering experimentally relevant ranges. Here, $L_{T_{\mathrm{i}}} \equiv-T_{\mathrm{i}} / \nabla T_{\mathrm{i}}, q$ is the safety factor, and $\hat{s}=\frac{\hat{\rho}}{q} \frac{\partial q}{\partial \hat{\rho}}$ the magnetic shear. The gradient in the expression for $L_{T_{\mathrm{i}}}$ is defined with respect to the mid-plane averaged minor radius of the flux surface. The parameter ranges in the training set are identical to those in [19], and are repeated in table 2. Also the training methodology was similar and for brevity not repeated here.

Only ITG modes were unstable throughout the parameter range of the training set, due to specific values of the input parameters that were kept fixed in the construction of the neural network trainings set. The instabilities found in the discharge simulated here are all in the ITG regime, as corroborated in [33] by an independent linear stability analysis for a similar discharge with nearly identical parameters. This increases the validity of this proof-of-principle version of the QLKNN transport model, when applied to this specific JET discharge. However for increased generality, work is in progress on neural-network regressions of QuaLiKiz including higher input dimensionality and cover ITG/TEM/ETG turbulence regimes [34, 35].

QLKNN4D-kin is 6 orders of magnitude faster than QuaLiKiz itself, predicting full profiles of transport coefficients on millisecond timescales, making it suitable for fasterthan-realtime tokamak simulation. One essential feature of this neural network-emulated transport model is that analytical derivatives can be obtained for the transport coefficients with respect to the plasma profiles, which allows for fully implicit solution of the transport equations (see appendix for details), with great advantages in numerical stability and speed.

\section{Simulation results}

\subsection{Simulation of JET shot \#73342 with $\psi, T_{e}, T_{i}$ and $n_{e}$}

3.1.1. Simulation setup. To demonstrate the multi-channel simulations, we will predict the core profiles of JET discharge \#73342 using RAPTOR and the QLKNN-4Dkin transport model. JET \#73342 is a C-wall baseline discharge with $B / I_{\mathrm{p}}=2.7 \mathrm{~T} / 2.5 \mathrm{MA}, 15 \mathrm{MW}$ of NBI heating and $2 \mathrm{MW}$ of ICRH power. The discharge has been analyzed in [33], and 

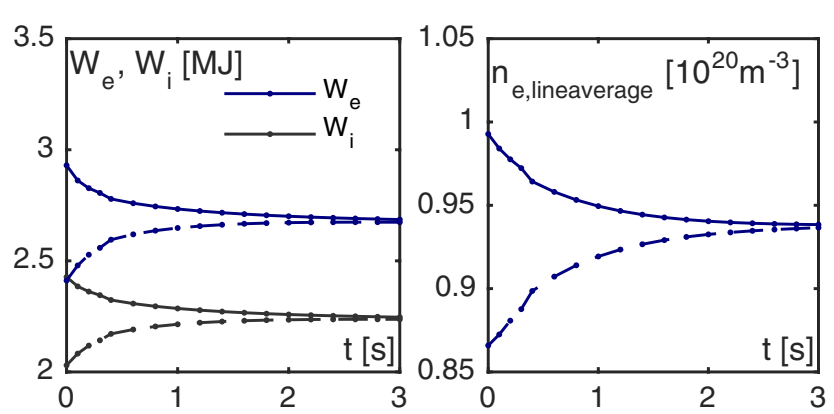

Figure 1. Time-convergence of electron and ion thermal energy $W_{\mathrm{e}}, W_{\mathrm{i}}$ and line integrated density during the RAPTOR simulation. The simulations are initialized with core profile values $20 \%$ higher/ lower than the final profiles of the CRONOS simulation, while the pedestal value is kept constant. The profiles converge to the same values after several energy and particle redistribution times.

the reader is referred to that work for further details on the discharge. The results are compared to experimental measurements averaged over $0.5 \mathrm{~s}$ in the middle of the flat-top phase of the discharge, from Charge Exchange $\left(T_{\mathrm{i}}\right)$ and high resolution Thomson scattering $\left(T_{\mathrm{e}}, n_{\mathrm{e}}\right)$ as well as to simulations using CRONOS with the same QLKNN-4Dkin transport model.

The CRONOS simulation was run for $2.5 \mathrm{~s}$ with boundary conditions at the pedestal top taken from time-averages of the experimental measurements. It evolves the profiles together with a self-consistent equilibrium from HELENA [36] and uses NEMO-SPOT [37] to calculate the NBI power/particle deposition, and driven current profiles. No line radiation, ICRH power or MHD effects were included in this simulation.

For the RAPTOR simulation, profiles of the power density to electrons and ions, the particle source profile, and the equilibrium, are taken directly from the final time of the CRONOS simulation, and kept constant. The profile of $Z_{\text {eff }}$ was set to a constant value of 1.8 as in CRONOS, which was determined using Bremsstrahlung measurements. The ion density $n_{\mathrm{i}}$ and of one impurity species (assumed to be carbon) were scaled based on the evolving $n_{\mathrm{e}}$ profile, constrained by quasineutrality and $Z_{\text {eff. }}$. The line-radiation profile was taken from bolometry data, and was observed to be dominated by edge radiation. ICRH profiles were calculated using the TORIC code [38] within TRANSP [39]. Sawtooth effects were modeled as described in section 2.2.3, where the critical magnetic shear at $q=1$ that triggers a sawtooth crash, was tuned to match the experimentallly observed sawtooth period of $\sim 10 \mathrm{~Hz}$ in this phase of the discharge. All these physics effects were considered when comparing the RAPTOR simulations to the experimental data. However, for the benchmark with the CRONOS simulation, the effects of line radiation, ICRH and sawteeth were not included, matching the setup of this particular CRONOS run.

The initial conditions for the RAPTOR simulation were set as follows: For the $q$ profile, we choose the profile at the end of the CRONOS simulation. For $T_{\mathrm{e}}, T_{\mathrm{i}}, n_{\mathrm{e}}$, we choose profiles $20 \%$ higher than the final profiles of the CRONOS simulation, to allow for discernible evolution of the profiles towards stationary state. The simulation is run for 2 JET seconds. Since this is larger than the energy confinement time $(<0.3 \mathrm{~s})$ and particle confinement time $(<1.0 \mathrm{~s})$, the particle and energy balances are close to their equilibrium. The current distribution time is, however, longer than the simulation time. If left to evolve further, the $q$ profile would change, affecting the transport fluxes via the dependency on $q$ and $s$. However, in the interest of comparing the results to the CRONOS results with a given $q$ profile, we choose to stop the simulation before the $q$ profile has evolved significantly. The obtained profiles are therefore not the true steady-state profiles, but the profiles corresponding to the $q$ profile given as input.

This is illustrated by the time evolution of the on-axis $T_{\mathrm{e}}$, $T_{\mathrm{i}}$, and $n_{\mathrm{e}}$ in the RAPTOR simulation shown in figure 1 . The values converge to their quasi-stationary values. In each plot, two time scales of profile evolution are evident. The fast (for $T$ and $n$ ) timescale, and a slower evolution associated with the resistive timescale due to the dependence of transport on the $q$-profile. The $n$ evolution timescale is slower by a factor $\sim 5$ with respect to the temperature evolution timescale.

\subsubsection{Benchmark between RAPTOR and CRONOS. The} RAPTOR simulation of JET 73342 is shown in figure 2, showing the quasi-stationary state profiles of $T_{\mathrm{e}}, T_{\mathrm{i}}$ and $n_{\mathrm{e}}$ following $2 \mathrm{~s}$ of profile evolution. The comparison between RAPTOR and CRONOS is excellent, with only minor deviations, attributed to the different methods for smoothing of the transport coefficients employed in RAPTOR and CRONOS. This verifies the implementation of the ion temperature and density transport equations in RAPTOR, as well as the coupling of QLKNN4D-kin.

The boundary condition in the simulations was set at $\hat{\rho}=0.85$, inside the (ELM-averaged) pedestal. The values of the profiles outside this position are of minor importance for core profile predictions, as the $q$ profile is not significantly affected since we simulate over times shorter than the current diffusion time. The mismatch of the $\hat{\rho}=1$ boundary condition (due to a lack of consistency in the $\chi$ values in that range) is hence not of importance to the comparisons.

Since the simulations shown in figure 2 did not include a sawtooth model, and since local gyrokinetic turbulence calculations tend to predict stability in the inner radii $(\hat{\rho}<0.2)$, which would lead to significant local peaking, the transport coefficients in the core region were adjusted to yield flat core profiles. This was carried out by imposing minimum values of the transport coefficients in the simulation. In this case $D_{\mathrm{e}, \min }=0.1 \mathrm{~m}^{2} \mathrm{~s}^{-1}, \chi_{\mathrm{e}, \min }=0.15 \mathrm{~m}^{2} \mathrm{~s}^{-1}$, and $\chi_{\mathrm{i}, \min }=$ $0.2 \mathrm{~m}^{2} \mathrm{~s}^{-1}$. Also, when $D_{\mathrm{e}}=D_{\mathrm{e}}$, min, then $V_{\mathrm{e}}=0$. Such a prescription effectively acts as a proxy for the effect of sawteeth.

\subsubsection{Comparison between RAPTOR and experimental} results. In figure 3, a comparison is shown between RAPTOR simulations and experimental measurements. In this case, the effects of line radiation, IC heating and sawteeth are simulated. Including these effects causes an increase in the region $\rho_{N}<0.3$ of only $\sim 10 \%$ in the sawtooth-averaged profiles. This relatively small difference means that we can employ prescribed minimum transport coefficients in the region affected by sawteeth as a proxy for their impact, allowing larger time 

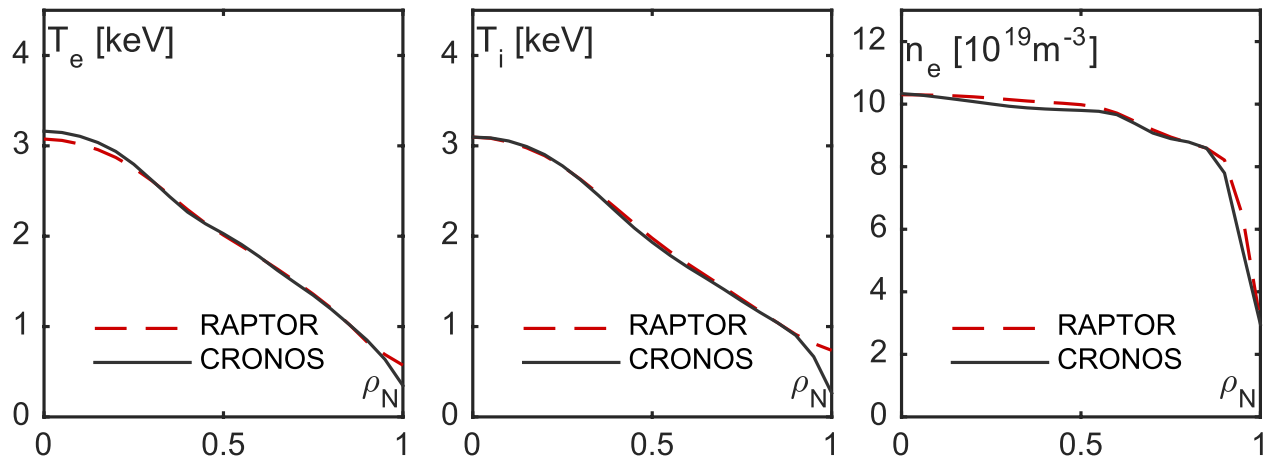

Figure 2. Comparison of RAPTOR profiles using QLKNN-4Dkin transport model to CRONOS simulations using the same transport model. Agreement is excellent except for the region outside $\rho_{N}>0.85$, owing to a different treatment of boundary conditions.
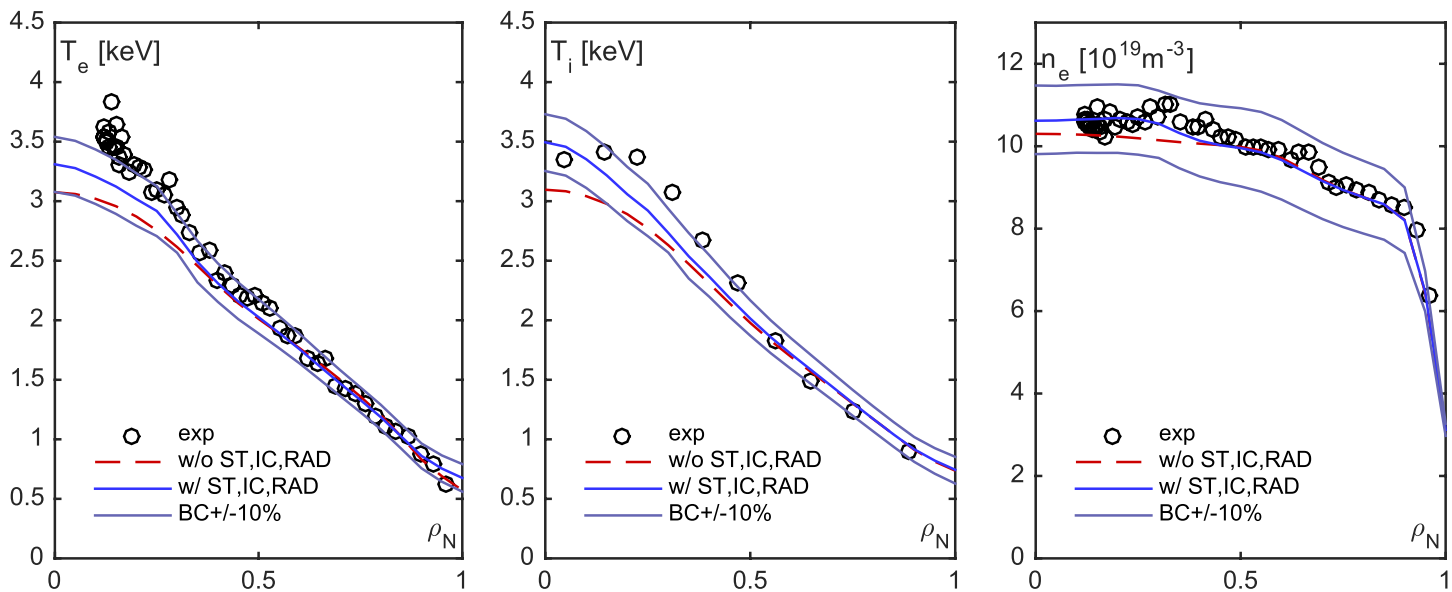

Figure 3. Comparison of RAPTOR profiles using QLKNN-4Dkin transport model and experimental data for JET shot 73342. Red-dashed curves show the results for the case without sawteeth, ICRH heating and line radiation, corresponding to the case shown in figure 2 . Bluesolid curves show the results after adding these effects. For the latter case, the boundary conditions at $\hat{\rho}=0.85$ are also perturbed by $\pm 10 \%$ for sensitivity analysis. Experimental statistical errors are estimated at $\pm 10 \%$.

Table 1. Standard deviation figures of merit for the RAPTORQLKNN4D-kin JET simulations shown in figure 3.

\begin{tabular}{lrrr}
\hline Case & \multicolumn{1}{c}{$\sigma_{T_{\mathrm{i}}}$} & \multicolumn{1}{c}{$\sigma_{T_{\mathrm{e}}}$} & \multicolumn{1}{c}{$\sigma_{n_{\mathrm{e}}}$} \\
\hline Nominal & $10.0 \%$ & $6.9 \%$ & $2.7 \%$ \\
BC reduced by 10\% & $13.1 \%$ & $10.6 \%$ & $11.0 \%$ \\
BC increased by 10\% & $4.0 \%$ & $6.5 \%$ & $7.1 \%$ \\
\hline
\end{tabular}

steps and faster simulations. We also show the effect of perturbing the boundary condition at $\rho_{N}=0.85$ by $10 \%$.

The agreement with experimental data is quantified in table 1, using a standard deviation figure of merit [40, 41], where, for a quantity $f$ :

$$
\sigma=\sqrt{\int_{\hat{\rho}_{\text {in }}}^{\hat{\rho}_{\mathrm{BC}}} \mathrm{d} x\left(f_{\mathrm{sim}}-f_{\mathrm{exp}}\right)^{2}} / \sqrt{\int_{\hat{\rho}_{\text {in }}}^{\hat{\rho}_{\mathrm{BC}}} \mathrm{d} x f_{\exp }^{2}} .
$$

Where $f_{\text {sim }}$ is the simulated quantity, and $f_{\exp }$ is the measured quantity. We have evaluated the figure of merit with $\hat{\rho}_{\text {min }}=0.2$, to avoid the sawtoothing region. We have included a propagation of the estimated boundary condition uncertainty in the comparison. The predicted and simulated profiles generally agree within $\sim 10 \%$, on the order of the measured statistical uncertainties. However, there is a slight systematic underprediction of the temperature gradients in the inner half-radius.

\subsection{Comparison of full Qualikiz versus QLKNN-4Dkin for simulation of JET \#73342}

The RAPTOR + QLKNN-4Dkin predictions in section 3.1 show good correspondence with the experimental measurements, in spite of the restricted dimensionality of the QuaLiKiz training set used for the neural network fitting. To further understand the sources of discrepancy, we performed a comparison between QLKNN-4Dkin, where many dimensionless quantites are assumed constant, and full QuaLiKiz. For simplicity, we restrict the analysis to a standalone transport coefficient comparison at two radial locations, at $\hat{\rho}=0.8$ where the gradient agreement is excellent, and at $\hat{\rho}=0.5$ where the ion and electron temperature gradients were underpredicted by QLKNN-4Dkin.

The dimensionless parameter inputs are shown in table 2 . The first row corresponds to the dimensionless parameters employed in the QuaLiKiz-4Dkin neural network training set. The values for $R / L_{T_{\mathrm{e}}}, R / L_{n_{\mathrm{e}}}, \epsilon, \alpha, \nu^{*}$ and $Z_{\mathrm{eff}}$ are shown, which are kept fixed in the training database. For the other values $\left(R / L_{T_{\mathrm{i}}}, T_{\mathrm{i}} / T_{\mathrm{e}}, q, \hat{s}\right)$, which were varied in the training set, we display their ranges. QuaLiKiz is an electrostatic code, hence $\beta$ is not one of the dimensionless parameters. The second and third row correspond to the full dimensionless parameter set 
Table 2. JET 733424 dimensionless parameters at 2 radial locations at $\hat{\rho}=0.5$ and $\hat{\rho}=0.8$, taken from the stationary profiles of the RAPTOR simulation displayed in figure 3. $\hat{\rho}$ is the normalized toroidal flux coordinate. $\nu^{*}$ is the normalized electron collisionality: $\nu^{*} \equiv \nu_{\mathrm{ei}} \frac{q R}{\epsilon^{1.5} v_{\mathrm{te}}}$, with $\epsilon=a / R, v_{\mathrm{te}} \equiv \sqrt{T_{\mathrm{e}} / m_{\mathrm{e}}}$ and $\alpha \equiv q^{2} \sum_{j} \beta_{j}\left(R / L_{n j}+R / L_{T j}\right)$. The dimensionless parameters are compared to the values used in the QuaLiKiz runs applied for the 4D neural network (NN) training set (1st row). The first four quantities, which can vary in the $\mathrm{NN}$ inputs, are marked as (min-max) indicating the minimum/maximum values in the database used to train the neural network. The $\nu^{*}$ in the first row corresponds to the small variation due to the varying $q$ in the training set, whereas the underlying $\nu_{\mathrm{ei}}$ remains constant-in any case $\nu^{*}$ remains in the near-collisionless regime. No rotation was included in the simulations considered here.

\begin{tabular}{lllllllllll}
\hline Data source & $R / L_{\mathrm{Ti}}$ & $T_{\mathrm{i}} / T_{\mathrm{e}}$ & $\hat{s}$ & $q$ & $R / L_{\mathrm{Te}}$ & $R / L_{n}$ & $\epsilon$ & $\alpha$ & $\nu^{*}$ & $Z_{\mathrm{eff}}$ \\
\hline QLKNN4D-kin & {$[2-12]$} & {$[0.3-3]$} & {$[0.1-3]$} & {$[1-5]$} & 6 & 2 & 0.167 & 0 & {$\left[1.3 \times 10^{-3}-6 \times 10^{-3}\right]$} & 1.0 \\
JET 73342 simulation $\hat{\rho}=0.5$ & 0.4 & 0.97 & 0.9 & 5.0 & 3.9 & 1.5 & 0.172 & 0.19 & 0.05 & 1.8 \\
JET 73342 simulaiton $\hat{\rho}=0.8$ & 1.5 & 0.96 & 1.7 & 8.5 & 10.8 & 2.6 & 0.275 & 0.71 & 0.17 & 1.8 \\
\hline
\end{tabular}
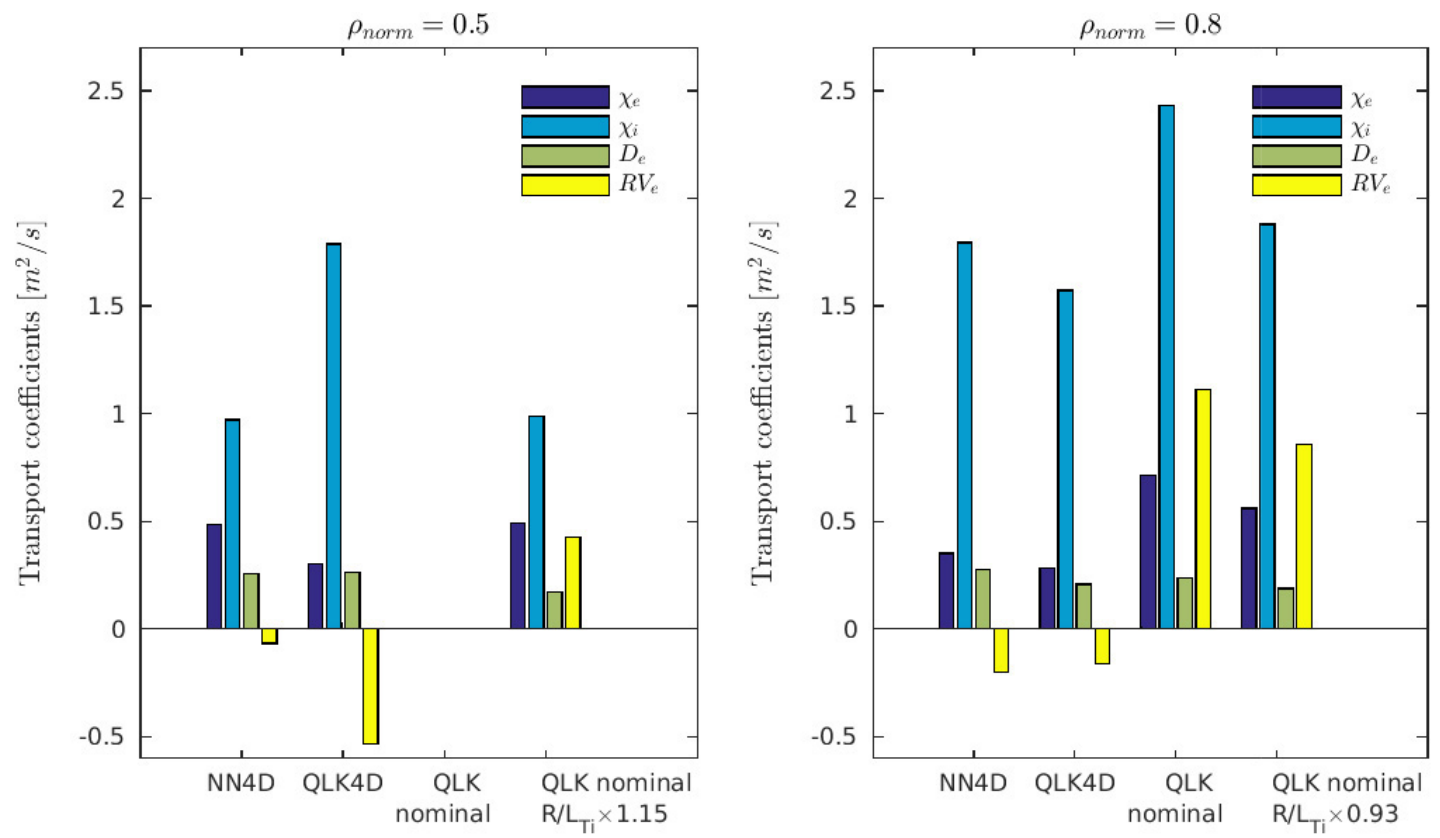

Figure 4. Comparison of transport coefficients between QLKNN4D-kin (NN4D) and full QuaLiKiz with the same fixed parameters as used for training the network (QLK4D), full QuaLiKiz with input parameters taken from the experiment, albeit without rotation (QLK nominal), and full QuaLiKiz with manually scaled $R / L T_{\mathrm{i}}$ to match experimental heat fluxes.

at $\hat{\rho}=0.5$ and $\hat{\rho}=0.8$ respectively, taken from the last time point in the nominal RAPTOR simulation.

The comparison between QLKNN-4Dkin and QuaLiKiz standalone calculations is shown in figure 4. The left panel corresponds to calculation at $\hat{\rho}=0.5$, and the right panel to $\hat{\rho}=0.8$. The values of the transport coefficients that are computed by the transport model $\left(\chi_{\mathrm{e}}, \chi_{\mathrm{i}}, D_{\mathrm{e}}, R V_{\mathrm{e}}\right)$ are shown for various calculations. All transport coefficients are in SI units, rescaled using the local plasma parameters at each radius from the QuaLiKiz-computed GyroBohm-normalized coefficients.

The set labelled 'NN4D' corresponds to the neural network transport model prediction. The set labelled 'QLK4D' corresponds to the QuaLiKiz calculated transport coefficients, where the fixed variables from the neural network training are imposed, and $R / L_{\mathrm{Ti}}, T_{\mathrm{i}} / T_{\mathrm{e}}, \hat{s}, q$ are all included with their nominal values. The comparison of NN4D and QLK4D indicates the quality of the NN regression of QuaLiKiz. It is evident that the regression provides a good match, particularly at $\hat{\rho}=0.8$, with some mismatch at $\hat{\rho}=0.5$. From additional analysis, it appears that these discrepancies, which result from imperfect neural network regression, can be resolved by $\sim 5 \%$ modifications of the input parameters, reflecting the large sensitivity of the transport fluxes to the inputs, a property often described as 'stiffness'.

The set labelled 'QLK nominal' corresponds to including all parameters taken from the nominal simulation profiles in the full QuaLiKiz calculation. This serves to analyze the impact of including the actual $R / L_{\mathrm{Te}}, R / L_{n}, \epsilon, \alpha, \nu^{*}$ and $Z_{\mathrm{eff}}$ on the resulting transport coefficients. For the $\hat{\rho}=0.5$ case, the unstable modes are stabilized (hence transport coefficients are zero). At $\hat{\rho}=0.8$, only a moderate increase in transport is predicted. This is surprising considering the significant differences in the parameters compared to the fixed training set values, particularly for $\alpha, \nu^{*}$ and $R / L_{T_{\mathrm{e}}}$. However, the destabilizing impact (e.g. of higher $\epsilon$ and $\alpha$ ) and stabilizing impact (e.g. of lower $\nu^{*}$ and higher $Z_{\mathrm{eff}}$ ) of the various parameters in this specific regime, tend to cancel each other out, coincidentally. Through an increase of $R / L_{T_{\mathrm{i}}}$ by $15 \%$ at $\hat{\rho}=0.5$, and a reduction by only $7 \%$ at $\hat{\rho}=0.8$, the original heat transport coefficients are recovered, as shown in the rightmost transport coefficient set. This suggests that the underprediction of the temperature 

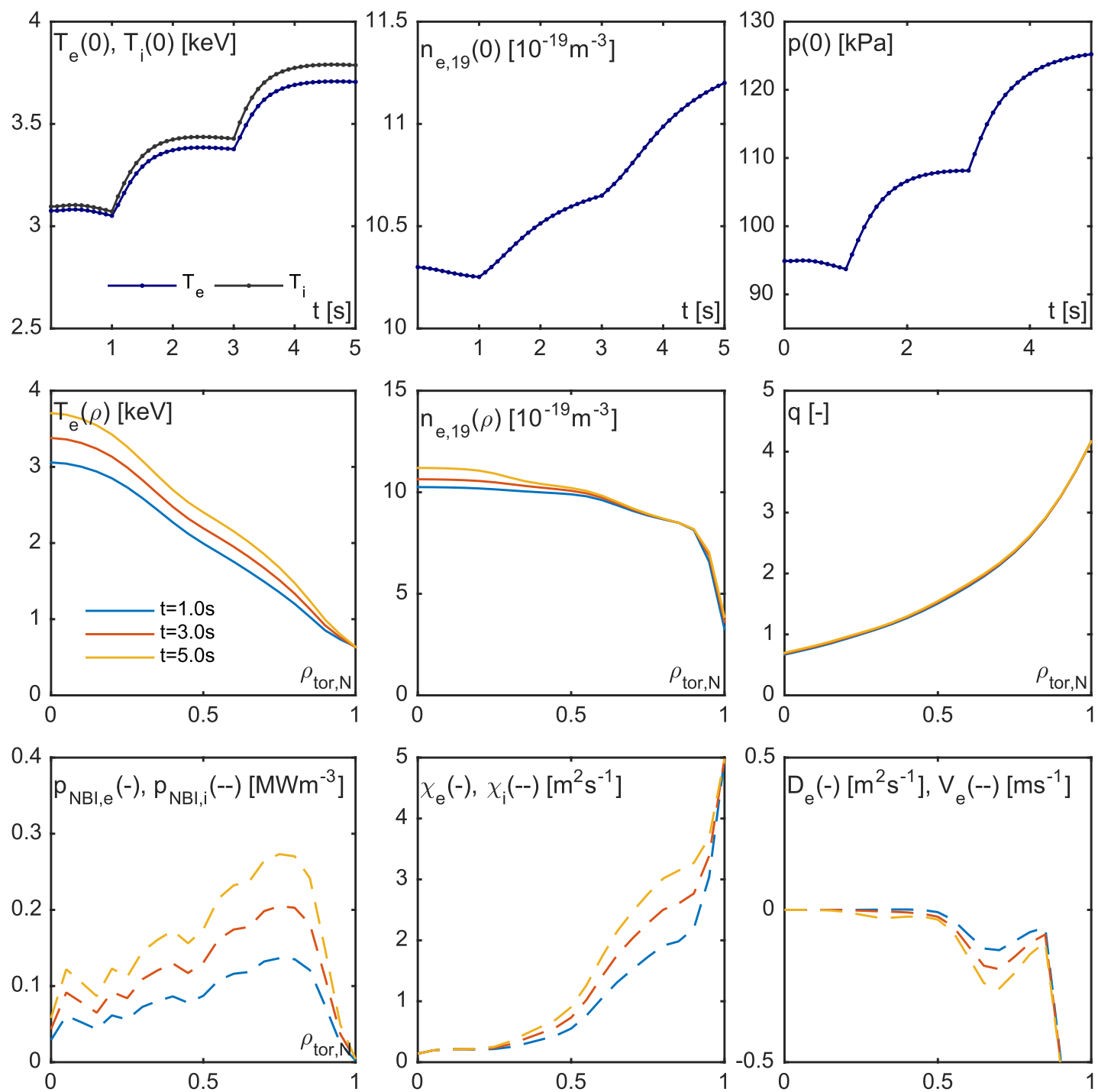

Figure 5. Time-dependent simulation using RAPTOR with QLKNN-4Dkin and a core-pedestal coupling model. Two steps in NBI power are simulated, resulting in different temperature, density and conductivity profiles. Time-traces of temperatures, electron density and pressure are shown in the top panels. Profiles of electron temperature, density, and safety factor $q$ are shown in the middle panels. $T_{\mathrm{i}}$ is omitted since its evolution strongly resembles that of $T_{\mathrm{e}}$. Sources, thermal conductivities and particle transport coefficients are presented in the bottom panels. The various colours correspond to the profiles at $t=1 \mathrm{~s}, t=3 \mathrm{~s}$ and $t=5 \mathrm{~s}$

gradients at mid-radius would be partially rectified by including all nominal parameters. The only discrepancy is in the particle convection term $R V_{\mathrm{e}}$, which reverses sign when including all nominal parameters, possibly indicating that the nominal QuaLiKiz prediction would lead to flatter density. However these effects are included in the $\mathrm{NN}$ regression of QLK under development [34, 35].

Furthermore, this analysis has confirmed that ITG modes are dominant in this regime, confirming the validity of QLKNN4D-kin for this specific case. In wider parameter regimes, where more mode classes may be unstable, a more general neural network emulation of QuaLiKiz will be used. Indeed, a 10D neural network emulation of QuaLiKiz, including $R / L_{\mathrm{Te}}, R / L_{n}, \epsilon, \nu^{*}, Z_{\text {eff }}$ and rotation, is currently under construction [34]. Additionally, recent versions of QuaLiKiz [16, 42] do include rotation and will be used to train future versions of the neural network emulations.

\subsection{NBI input power scan including core-pedestal coupling}

To conclude this section, we present demonstrative timedependent simulations of an NBI power ramp based on the discharge presented in section 3.1.2 (without sawteeth). Starting from the final profiles of that simulation, a $5 \mathrm{~s}$ simulation is carried out with two steps of NBI power. These steps in power are simulated by scaling the NBI heat and particle sources, and driven current profile, by $\times 1.5$ (between $1 \mathrm{~s}$ and $3 \mathrm{~s}$ ) and $\times 2$ (from $3 \mathrm{~s}$ until $5 \mathrm{~s}$ ) respectively. This is not an entirely accurate representation of the true effect of increasing NBI power, which would need to consider a self-consistent evaluation of the ionization and fast ion slowing-down physics with respect to the evolving plasma parameters.

In order to simulate the expected increase in pedestal pressure with increasing power, a simple model for the boundary condition is introduced that couples the pedestal top temperature to the core profiles via proportionality to the Shafranov 

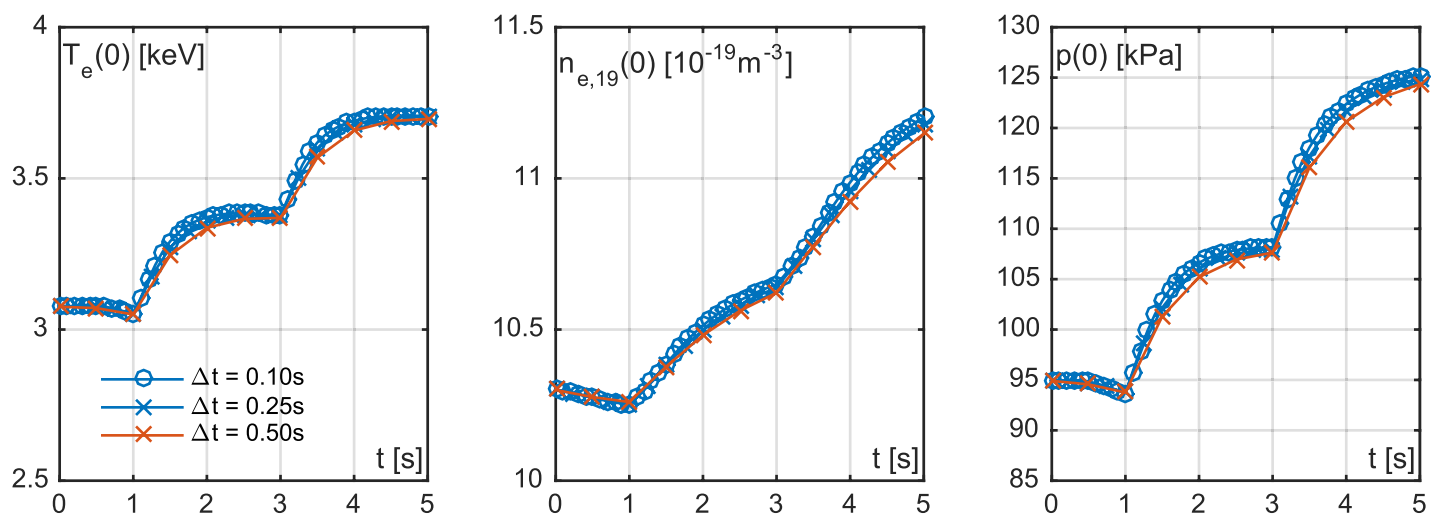

Figure 6. Comparison of time-dependent simulations as shown in figure 5, executed with different simulation time steps. Owing to the numerical stability of the fully implicit time scheme used by RAPTOR, the simulation is numerically stable even for very large time steps up to $0.5 \mathrm{~s}$ (which is longer than a JET confinement time). For such large time steps, the transient phase following the power steps can not be captured accurately, while the simulations converge to similar profiles on longer time scales.

shift parameter: $\Delta_{\mathrm{s}}=\beta_{\mathrm{p}}+l_{\mathrm{i}} / 2$. Pedestal confinement is known to improve with Shafranov shift, through an increased critical threshold of peeling-ballooning modes [43]. This simple, ad-hoc model is written (both for ion and electron temperature) as

$$
T_{\mathrm{e}, \mathrm{i}}\left(\rho_{\mathrm{ped}}\right)=c_{T_{b}}\left(\Delta_{\mathrm{s}}-\Delta_{\mathrm{s}}^{0}\right)+T_{b 0} .
$$

The values of $\Delta_{\mathrm{s}}^{0}, T_{e b 0}$ are taken from the initial condition of the simulation. The free parameter $c_{T_{e b}}$ is chosen to maintain the $H_{98}$ factor roughly constant during the power scan.

Results of the simulation, which took only a few seconds to execute on single $2.6 \mathrm{GHz}$ Intel ${ }^{\mathrm{R}}$ core $^{\mathrm{TM}}$ i5 processor, are shown in figure 5. On the top panels, the time-history of the central temperatures, electron density and pressure are shown. Clearly, two time scales can be seen owing to the (fast) confinement times and (slow) current redistribution times. Kinetic and source profiles at the initial, middle and final time of the simulation are shown in the middle and lower panels.

The changes in the pedestal boundary condition at $\rho_{\text {ped }}=0.85$ are clearly visible as contributing to the increase in temperature. We emphasize that the variation in pedestal boundary condition is due to the response of the firstprinciple-based core transport model to the power increment. Also, the values in the center region $(\hat{\rho}<0.2)$ are sensitive to the prescribed minimum values of the transport coefficients that are used in regions where ITG modes are stable. The $q$ changes are rather small owing to the long global current redistribution time for JET discharges at these temperatures (typically $\sim 10 \mathrm{~s}$ ). The values of $H_{98}$ are $(0.796,0.7560 .755)$ at $t=[1,3,5]$ respectively.

Note that while it is known that in reality $H_{98}$ tends to increase with power at JET [44], (owing an the overly pessimistic $P^{-0.7}$ term in the $H_{98}$ scaling), the purpose of this model is to demonstrate the core-pedestal coupling in these time-dependent simulations. In the future, this capability can be used to include a more physics-based pedestal model into these simulations, for example as in [20].

Finally, as a demonstration of the numerical stability of the code, we repeat the simulations shown in figure 5, which were done with time steps of $0.1 \mathrm{~s}$, with time steps of $0.25 \mathrm{~s}$ and 0.5 s respectively. As can be witnessed from figure 6, the results on long timescales are practically identical, while on time scales shorter than the confinement time, taking a too long time step results in some inaccuracy during the initial response to the power steps. Still, when only long-term time evolution is important, taking long time steps allows even faster simulations to be carried out.

\section{Conclusion and outlook}

In this paper, we have shown the extension of the RAPTOR control-oriented transport simulator to provide, for the first time, real-time capable simulations of core $T_{\mathrm{e}}, T_{\mathrm{i}}, n_{\mathrm{e}}$ and $q$ profiles in a tokamak.

These simulations presented here used the QLKNN-4Dkin transport model, providing first-principle predictions of the core profiles. By prescribing only the boundary conditions at the top of the H-mode pedestal, good agreement with JET experimental data was found in the region $\hat{\rho}<0.85$. Within $\hat{\rho}=0.5$, slightly lower core temperatures were obtained with respect to diagnostic measurements, which could be explained by the restricted input dimensionality of the present version of the QLKNN transport model. Work is in progress to extend the QuaLiKiz database to higher input dimensionality (10D), covering ITG, TEM, and ETG turbulence in a wider range of experimentally relevant input parameters, and to train new neural network transport models using these databases [34]. Furthermore, a JET-specific database spanning 14 QuaLiKiz input dimensions is being constructed, with data points clustered around regions of the parameter space that appear in experimental profiles based on 2000 discharges [35]. A multimachine extension thereof is also planned.

While here the new capabilities of RAPTOR to solve several transport equations have been illustrated using the QLKNN-4D transport model, other transport models can be used, e.g. Bohm-gyroBohm [31] or the model used in [4].

Further work on improving and extending the RAPTOR code for predictive simulation purposes will focus on coupling to free- and fixed-boundary Grad-Shafranov equlibrium solvers, as well as on fast models to estimate the sources and 
sinks of power and particles (including radiation). Furthermore, use of core-pedestal models is envisaged, for example using a neural network regression of pedestal heights and widths derived from the EPED model [45], as in [20]. Coupling to the edge would require validated, reduced models of the plasma in that region (e.g. 2-point models) or neural network regressions of more complex, higher dimensional models.

Finally, real-time applications of the new capabilities of RAPTOR are envisaged, for example for real-time monitoring of the discharge evolution with respect to increasingly accurate first-principle-based transport model predictions, allowing a control system to take appropriate action if discrepancies are detected. The addition of particle density transport equations enables, in particular, estimation of approaching density limits and prediction of impurity accumulation. Also, automated off-line tokamak scenario optimization as in [2-4] will benefit from more enhanced predictive capability of the transport simulator.

\section{Acknowledgments}

The work has been carried out within the framework of the EUROfusion Consortium and has received funding from the Euratom research and training programme 2014-2018 under grant agreement No 633053. The views and opinions expressed herein do not necessarily reflect those of the European Commission. This work was also supported in part by the Swiss National Science Foundation.

\section{Appendix. Details of the numerical implementation}

Details of the numerical implementation for the original RAPTOR code were given in [2] and [4]. The details of the implementation are updated here for completeness.

First, the PDE to be solved for a dependent variable $y(\hat{\rho}, t)$ is written in the generic form

$$
m_{y} \frac{\partial y}{\partial t}=\frac{\partial}{\partial \hat{\rho}}\left(a_{y} y\right)+\frac{\partial}{\partial \hat{\rho}} d_{y} \frac{\partial y}{\partial \hat{\rho}}+h_{y} T_{\mathrm{e}}+s_{y}
$$

where $m_{y}, a_{y}, d_{y}, h_{y}, s_{y}$ may also depend on $(\rho, t)$.

Then, the dependent variable is expressed as a sum of $n_{s p}$ third-order B-spline basis functions: $y(\hat{\rho}, t)=\sum_{\mathrm{i}}^{n_{s p}} \Lambda_{\alpha}(\rho) \hat{y}_{\alpha}$ with (scalar) basis function coefficients $\hat{y}_{\alpha}$. The set of basis functions is chosen so as to identically satisfy the boundary condition at $\rho=0$. The resulting equation is multiplied by a set of trial functions $\Lambda_{\beta}$, chosen equal to $\Lambda_{\alpha}$. The result is integrated over the domain $\hat{\rho}=[0,1]$, where integration by parts is used to remove second-order derivative terms, at the expense of introducing additional boundary terms.

This yields a set of nonlinear ordinary differential equations, written in matrix-vector form as:

$$
\mathbf{M}_{y} \dot{\hat{\mathbf{y}}}=\left(-\mathbf{A}_{y}-\mathbf{D}_{y}+\mathbf{H}_{y}\right) \hat{\mathbf{y}}+\mathbf{l}+\mathbf{s}
$$

where the $\alpha, \beta$ elements of the matrices are:

$$
\mathbf{M}_{y,(\alpha, \beta)}=\int_{0}^{1} m_{y} \Lambda_{\beta} \Lambda_{\alpha} \mathrm{d} \hat{\rho}
$$

$$
\begin{gathered}
\mathbf{A}_{y,(\alpha, \beta)}=\int_{0}^{1} a_{y} \frac{\partial \Lambda_{\beta}}{\partial \hat{\rho}} \Lambda_{\alpha} \mathrm{d} \hat{\rho} \\
\mathbf{D}_{y,(\alpha, \beta)}=\int_{0}^{1} d_{y} \frac{\partial \Lambda_{\beta}}{\partial \hat{\rho}} \frac{\partial \Lambda_{\alpha}}{\partial \hat{\rho}} \mathrm{d} \hat{\rho} \\
\mathbf{H}_{y,(\alpha, \beta)}=\int_{0}^{1} h_{y} \Lambda_{\beta} \Lambda_{\alpha} \mathrm{d} \hat{\rho} .
\end{gathered}
$$

Furthermore

$$
\begin{gathered}
\mathbf{s}_{y, \beta}=\int_{0}^{1} \Lambda_{\beta} s_{y} \mathrm{~d} \hat{\rho} \\
\mathbf{l}_{y, \beta}=\left[\left(a_{y} y+d_{y} \frac{\partial y}{\partial \hat{\rho}}\right) \Lambda_{\beta}\right]_{\hat{\rho}=1} \text { if } \beta=n_{s p}, \quad 0 \text { otherwise. }
\end{gathered}
$$

Note that there are many nonlinearities in (A.2) due to the possible dependence of $m_{y}, a_{y}, d_{y}, h_{y}, s_{y}$ on all the profiles that are being solved for.

In this form, the matrix $\left(-\mathbf{A}_{y}-\mathbf{D}_{y}+\mathbf{H}_{y}\right)$ does not have full rank, reflecting the fact that we need to impose a boundary condition to solve the PDE. To this end, the last equation of (A.2) is replaced by an equation imposing the boundary condition, written as $\sum_{\alpha} \Lambda_{\alpha}\left(\hat{\rho}_{\mathrm{BC}}\right)=y_{\mathrm{BC}}$. In this way, it is possible to impose time-varying boundary conditions at a time-varying radial location, which is useful when switching from L-mode (where one might prescribes the boundary condition at $\hat{\rho}=1$ ) to H-mode (where one might prescribe the boundary condition inside the pedestal top (e.g. $\hat{\rho}=0.85)$ ). It is also possible to set $y_{\mathrm{BC}}$ as being a function of the plasma profiles, in order to implement core-pedestal coupling.

The user can specify for which channels to solve the PDE. For each of these we obtain a system of ODEs for the vector $\mathbf{y}_{j} \forall j=\{1, \ldots, N\}$, where $N$ is the number of PDEs to be solved. By combining these into a single 'state' vector $x=\left[\mathbf{y}_{1}^{T}, \ldots, \mathbf{y}_{N}^{T}\right]^{T}$, the system of all the ODEs can be combined into a single equation of the form:

$$
0=\tilde{f}(\dot{x}(t), x(t), z(t))
$$

where we have collected all external time-dependent quantities that influence the PDE evolution (sources, transport coefficients, etc) into the variable $z(t)$.

As explained in more detail in [2], this continuous-time nonlinear ODE is discretized in time by a backward Euler method: $\dot{x}=\left(x_{k+1}-x_{k}\right) / \Delta t, x=x_{k+1}$. This yields the nonlinear equation:

$$
0=f\left(x_{k+1}, x_{k}, z_{k}\right) .
$$

With given $x_{k}$ (from the previous time step) and $z_{k}$, this can be solved for $x_{k+1}$. The solution is sought by Newton-Rapson iterations, where the required Jacobian $\frac{\partial f}{\partial x_{k+1}}$ is calculated analytically at each iteration. While it is cumbersome to implement these analytical Jacobians for the many nonlinear expressions that enter into tokamak transport coefficients and sources, this method of solution allows large time-steps to be taken (even exceeding the energy confinement time). 
RAPTOR is entirely implemented in MATLAB. For realtime applications, the code is included in a Simulink ${ }^{\mathrm{R}}$ model and C-code is automatically generated and compiled for the target real-time system [46]. For stand-alone use, MatLaB code can also be automatically converted to C-code and compiled, also providing a significant speed-up for the purpose of optimization or systematic scans which may require many runs. The latter option was used to determine the execution times cited in this paper.

\section{ORCID iDs}

F. Felici (1) https://orcid.org/0000-0001-7585-376X

J. Citrin (1) https://orcid.org/0000-0001-8007-5501

A.A. Teplukhina (1) https://orcid.org/0000-0002-9213-7594

O. Sauter (1) https://orcid.org/0000-0002-0099-6675

\section{References}

[1] Humphreys D. et al 2015 Phys. Plasmas 22021806

[2] Felici F. et al 2012 Plasma Phys. Control. Fusion 54025002

[3] van Dongen J. et al 2014 Plasma Phys. Control. Fusion 56125008

[4] Teplukhina A.A. et al 2017 Plasma Phys. Control. Fusion 59124004

[5] Vu N.M.T. et al 2016 Control Eng. Pract. 5434

[6] Maljaars E. et al 2017 Nucl. Fusion 57126063

[7] Artaud J. et al 2010 Nucl. Fusion 50043001

[8] Ou Y. et al 2007 Fusion Eng. Des. 821153

[9] Felici F. et al 2014 American Control Conf. (Portland, OR: IEEE) pp 4816-23

[10] Felici F. et al 2011 Nucl. Fusion 51083052

[11] Felici F. et al 2016 Preprint: 2016 IAEA Fusion Energy Conf. (Kyoto) [EX/P8-33] (https://nucleus.iaea.org/sites/ fusionportal/Shared\%20Documents/FEC\%202016/fec2016preprints/preprint0519.pdf)

[12] Piron C. et al 2017 Fusion Eng. Des. 123 616-9

[13] Jenko F. et al 2000 Phys. Plasmas 71904

[14] Candy J. et al 2003 J. Computat. Phys. 186545

[15] Bourdelle C. et al 2016 Plasma Phys. Control. Fusion 58014036
[16] Citrin J. et al 2017 Plasma Phys. Control. Fusion 59124005

[17] Staebler G. M. et al 2013 Phys. Rev. Lett. 110055003

[18] Staebler G. M. et al 2007 Phys. Plasmas 14055909

[19] Citrin J. et al 2015 Nuclear Fusion 55092001

[20] Meneghini O. et al 2017 Nucl. Fusion 57086034

[21] Poli E. et al 2001 Comput. Phys. Commun. 13690

[22] Reich M. et al 2015 Fusion Eng. Des. 10073

[23] Weiland M. et al 2018 Nucl. Fusion (https://doi. org/10.1088/1741-4326/aabf0f)

[24] Sauter O. et al 2013 Comput. Phys. Commun. 184293

[25] Sauter O. et al 1999 Phys. Plasmas 62834

[26] Sauter O. et al 2002 Phys. Plasmas 95140

[27] Hinton F.L. et al 1976 Rev. Mod. Phys. 48239

[28] Porcelli F. et al 1996 Plasma Phys. Control. Fusion 382163

[29] Turri G. et al 2008 Fusion Energy ((Proc. 22nd Int. Conf. (Geneva, 2008)) (Vienna: IAEA) CD-ROM file EX/P and www-naweb.iaea.org/napc/physics/FEC/FEC2008/html/ index.htm)

[30] Basiuk V. et al 2017 Plasma Phys. Control. Fusion 59125012

[31] Erba M. et al 1998 Nucl. Fusion 381013

[32] Haykin S. 1999 Neural Networks: a Comprehensive Foundation 2nd edn (Englewood Cliffs, NJ: Prentice Hall)

[33] Baiocchi B. et al 2015 Plasma Phys. Control. Fusion 57035003

[34] van de Plassche K. et al 2017 44th EPS Conf. on Plasma Physics (Belfast, UK, 26-30 June 2017) p P2.182 (http:// ocs.ciemat.es/EPS2017PAP/pdf/P2.182.pdf)

[35] Ho A. et al 2017 44th EPS Conf. on Plasma Physics (Belfast, UK, 26-30 June 2017) $p$ P5.173 (http://ocs.ciemat.es/ EPS2017PAP/pdf/P5.173.pdf)

[36] Huysmans G. et al 1991 Europhysics Conf. on Computational Physics (Amsterdam, NL 371)

[37] Schneider M. et al 2011 Nucl. Fusion 51063019

[38] Brambilla M. 2002 Plasma Phys. Control. Fusion 442423

[39] Budny R.V. et al 2008 Nucl. Fusion 4875005

[40] ITER Physics Basis Expert Groups on Confinement, Transport, Confinement Modelling, Database and ITER Physics Basis Editors 1999 Nucl. Fusion 392175

[41] Holland C. 2016 Phys. Plasmas 23060901

[42] Cottier P. et al 2014 Plasma Phys. Control. Fusion 56015011

[43] Garcia J. et al 2015 Nucl. Fusion 55053007

[44] Challis C. et al 2015 Nucl. Fusion 55053031

[45] Snyder P.B. et al 2012 Phys. Plasmas 1956115

[46] Felici F. et al 2014 41st EPS Conference on Plasma Physics (Berlin, Germany) (http://ocs.ciemat.es/EPS2014PAP/pdf/ P2.002.pdf)

[47] Litaudon X. et al 2017 Nucl. Fusion 57102001

[48] Meyer H. et al 2017 Nucl. Fusion 57102014 